\title{
Service-Oriented Logistics Management System based on Internet of Things
}

\author{
Biao Dong ${ }^{1, \text { a }}$ \\ ${ }^{1}$ Nanjing Institute of Industry Technology, Nanjing, China \\ adongb@niit.edu.cn
}

\begin{abstract}
Keywords: middleware; wireless sensor networks; platform software
Abstract. Logistics information management system involves logistics, industry, commerce, and government department. The emergence of the Internet of Things(IOT) efficiently supports the construction of the logistics management system. Service-oriented paradigm is a suitable paradigm for IOT model. This paper designs SLMSIOT, a service-oriented logistics management system based on IOT, whose architecture is composed of application layer, logical sensor layer, and physical layer. Application layer provides customization tools to customize logistics business. Logical sensor layer is a bridge that connects the application layer with the physical world. It's divided into three main parts: service management, standard service, and logical sensor management. Physical layer includes a variety of devices.
\end{abstract}

\section{Introduction}

Automation, standardization, and intelligence are the most important character of modern logistics today. Information technology has become an important means to improve the service levels of the logistics enterprises, increase competitive advantage, and meet customer demand[1]. Logistics information management system involves logistics, industry, commerce, government department, etc. Computer, communication and network technology improve the processing and transfer speed of logistics information. The efficiency of logistics activities and rapid response capability are improved[2]. IOT is a development target of next generation Internet, and realizes the interconnection between things. The emergence of the IOT may efficiently support the construction of the logistics management system[3].

In terms of logistics management systems based on IOT, people have done a lot of work[4,5], but there are still some problems to be solved. In the aspect of technical application, most systems only study the application of one of the key technologies of IOT in the logistics management system, yet run short of systemic research on application of IOT's technical system in logistics management system. In the aspect of business application, most systems only propose solutions to a single problem in logistics management, yet run short of research on the entire logistics business chain.

This paper presents the model and system design that tailor SLMSIOT to IOT environment. Specifically, it makes the following primary contributions.

(1) Propose service-oriented logistics management system based on IOT, and define its architecture.

(2) Design and implement standard service, service management, and application layer module.

\section{SLMSIOT model}

Traditional logistics management systems have been unable to meet the needs of the business. Taking into account the rapid development of cloud computing and the IOT, we propose a service-oriented solutions based on IOT. Through a series of common services and a set of configuration tools, we only need to use customization tools to customize business. That is, we first combine the corresponding standard services to adapt their business processes, and upload to the cloud services; second, SLMSIOT automatically analyzes and validate business processes, and forms the corresponding data flow and the logical sensor processes; finally, By requesting access to standard equipments or 
accessing via the cloud with devices, we can achieve business process deployment. Service-oriented characteristic based on relied fields of study, such as reusability, configurability, customizability etc., is one of the important characteristics of SLMSIOT. The specific components, model and interface constitute SLMSIOT's architecture shown in Fig.1.

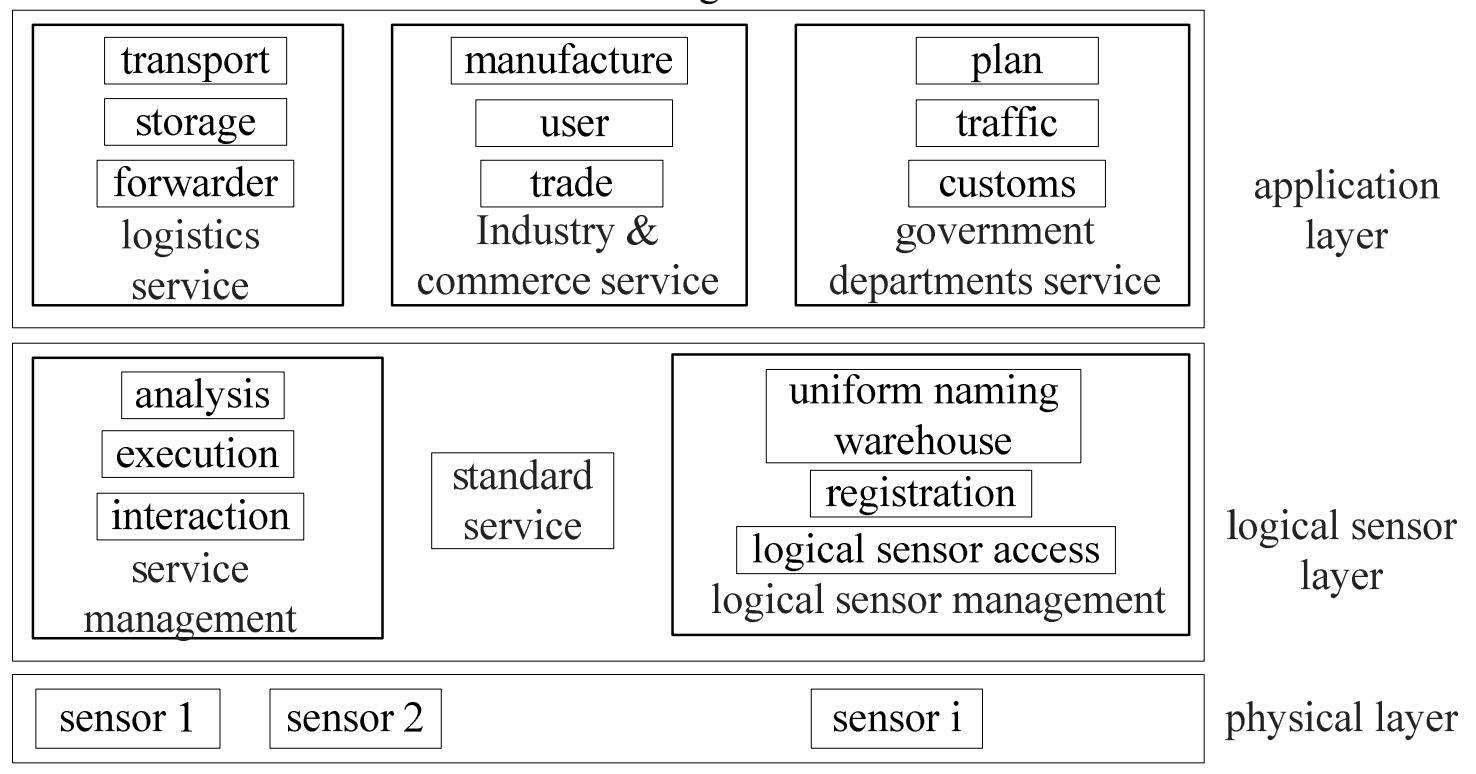

Fig.1. Reference model of SLMSIOT

SLMSIOT solution includes three layers: application layer, logical sensor layer, and physical layer. Application layer provides customization tools to customize business, which is divided into three main parts: logistics service, Industry \& commerce service, and government department service. Logical sensor layer is a bridge that connects the application layer with the physical world. It's divided into three main parts: service management, standard service, and logical sensor management. Physical layer includes a variety of devices, such as RFID reader, temperature sensor and pressure sensor. By abstraction, these devices are connected to SLMSIOT, and are managed as resources.

\section{SLMSIOT system design}

Standard service. After analyzing business in logistics, we extract the abstract sense of the business to form standard services. So the standard service itself is a workflow that contains the corresponding objects, events, and relationships between objects and events. Standard service can run independently and have a complete set of rules. Service flow is represented in the form of process nodes, and is customized by users in the form of plug-ins. We use Petri Nets for modeling service flow, and extract the common application processes. In order for users to customize business, application processes are mapped to service flow models, which are composed of standard services.

Standard service has a set of complete event handling mechanism, its events are divided into two types, namely primitive events and complex events. The primitive event is generally RFID tag event, including time, place, data content and other information. The complex event is composed of several primitive events or other complex events, and has certain characteristics of data flow, i.e., there is a certain logical sense between the events.

In this paper, the RFID event is the standard ALE event. Usually, ALE events are original RFID events, which don't have flow relations. By the event handling mechanism, we further handle ALE events. The mechanism describes complex events by like-SQL language, filters and extracts primitive events, and then combines the primitive events into a complex event. After parsing service profiles, the event handling mechanism extracts the standard service events, and registers to form services. By subscription and publication of the standard service events and the ALE events, the entire services complete the communication. 
Service management. Service management is responsible for the execution of the service flow. From the perspective of traditional workflow, it is a workflow engine. Service management must first solve the four issues, namely definition, scheduling, execution and instantiation, which need to be addressed by traditional workflow engines. In this paper, the service flow is composed of standard service nodes. Compared with traditional workflow nodes, the standard service nodes are with greater granularity. Because of the enormous differences of the standard services, service management must support user-defined process nodes, and is easy to manage and expand.

Service analysis module is responsible for verification, analysis, and instantiation of the service flow profiles. Its purpose is to resolve service flow definition model to service flow operation model.

Service execution module is responsible for execution from a service node to another node orderly. Its purpose is as follows: Support the service flow development language and its implementation; Support the basic operations of the service flow, such as running, management, monitoring, exception handling; Support to interact with peripheral systems, the module can be driven and queried by peripheral systems.

During the execution of the service flow, standard services run as an autonomous unit. Service execution module must be able to trigger the standard services, obtains the operation results from the standard services, and promotes the evolution of service flow. Therefore, service management must be able to communicate with the standard services, and provides interfaces for their calls. When users have customized a service flow, service management accepts the service flow and forms service flow objects. Service object is composed of service flow head node and the related standard service nodes. Each standard service node is driven by a specific RFID event. Service analysis module reports the performing signals of service flow to the service execution module. The service execution module changes the state of the service flow node.

Application layer. We attach RFID tags in the goods packaging, and install sensor devices in all locations involved in logistics. Application layer identifies and obtains real-time basic information, state information in logistics, and process information. By IOT standard language, the layer gathers and transports data that have been collected and perceived. We propose a reference framework, shown in Fig.2, to describe the IOT model of logistics management system integrated RFID and WSNs.

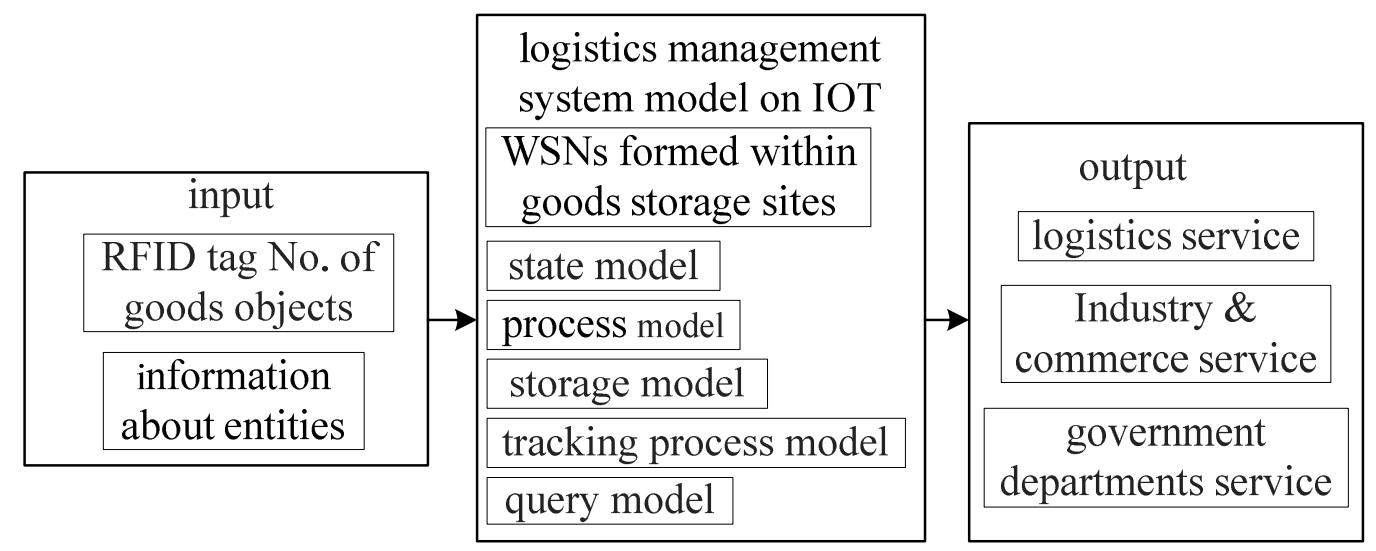

Fig.2. IOT model of logistics management system integrated RFID and WSNs

Definition 1 SLMSIOT. SLMSIOT can be represented by 4-tuple, and denoted as SLMSIOT= (configPML, Input, Output, ST). The meanings are as follows: configPML represents SLMSIOT's configuration files, describes the related parameters of logistics information, such as basic attributes, time, location, user-generated business documents, and perceived environmental information. Input represents SLMSIOT's input, describes RFID tag No. of goods objects and information about entities. Output represents SLMSIOT's output, provides these services, such as logistics service, Industry \& commerce service, and government departments service. ST represents the stored templates, describes the structure of the stored information, such as users, types of business, states and processes. 
In the paper, Let $S=\left\{S_{1}, S_{2}, \ldots, S_{n}\right\}$ denote the set of all goods entity objects, $S$ is either a piece of goods, or is a packing box or a packing crates, which is composed by a group of goods. Attr(S) indicates that the basic attribute values of $\mathrm{S}$, which is provided by the manufacturer of goods, including the compositions of goods, production time, packaging specifications, production batch number, production time and expiration date. Symbol $t$ represents the time when the state variation of $\mathrm{S}$ occurs, and is automatically generated when RFID readers read tags of S. Id(S) denotes the RFID tag No. of S. Because S may be single goods, a packing box, or a packing crates, so its corresponding tag number has also been distinguished. Loc(S) shows the location where the location variation of $\mathrm{S}$ occurs, and can be acquired by the ID of RFID reader. Envi(s) represents the current environmental information of $\mathrm{S}$, which is obtained by the sensors laid out on storing locations of $\mathrm{S}$, including temperature, humidity, light, etc.. By sensor networks, the obtained information is passed to sink nodes laid out on the location, and then, the information is passed to remote server via the Internet or in any other way. User(S) denotes the users resulting in the state of the $\mathrm{S}$ change, the users may be businesses, logistics companies, government agencies and end-users. Task(S) represents the business types of the S occurring in logistics process. Rela(S) represents RFID tag number of the entity objects associated with the $\mathrm{S}$. If $\mathrm{S}$ is a piece of goods, $\operatorname{Rela}(\mathrm{S})$ is the packing box RFID tag number of the $\mathrm{S}$. If $\mathrm{S}$ is a packing box, Rela(S) is the packing crates RFID tag number of the packing box. Ord(S) denotes the No. of business documents corresponding to the S, such as transport orders, invoices, and implements association with enterprise entity business data.

Definition 2 state. The state information of the $\mathrm{S}$ can be represented by 9-tuple, and denoted as $\mathrm{ST}(\mathrm{S}, \mathrm{t})=(\operatorname{Attr}(\mathrm{S}), \mathrm{t}, \operatorname{Id}(\mathrm{S}), \operatorname{Loc}(\mathrm{S}), \operatorname{Envi}(\mathrm{s}), \operatorname{User}(\mathrm{S})$, Task(S), Rela(S), Ord(S)), where ST(S,t) represents the state information of the $S$ when the $S$ circulates via a given place at the time $t$.

Definition 3 process. The process information of the $\mathrm{S}$ can be represented by 5-tuple, and denoted as $\operatorname{PR}(\mathrm{S})=(\operatorname{Attr}(\mathrm{S}), \operatorname{Loc}(\mathrm{S}), \operatorname{User}(\mathrm{S}), \operatorname{Task}(\mathrm{S}), \operatorname{Ord}(\mathrm{S}))$.

\section{Conclusions}

In this paper, we propose the logistics management system based on IOT. SLMSIOT model is defined in a service-oriented logistics environment. We illustrates a conclusion through SLMSIOT system design. The results show that SLMSIOT can easily be constructed.

\section{Acknowledgements}

This work was sponsored by Qing Lan project(Jiangsu province, china) and open fund project of Jiangsu provincial research and development center of intelligent sensor network engineering technology, china(ZK13-02-03, Software technology, platform and application for sensor network).

\section{References}

[1] D.Prajogo, J.Olhager: International Journal of Production Economics, Vol.135(1)(2012), p.514522.

[2] B.C.Ha, Y.K.Park, S.Cho: International Journal of Operations \& Production Management, Vol.31(1) (2011), p.56-77.

[3] L.Foschini, T.Taleb, A.Corradi, et al.: Communications Magazine, IEEE, Vol.49(11) (2011), p.50-57.

[4] B.Karakostas: Procedia Computer Science, Vol.19 (2013), p.594-601.

[5] K.Hribernik, T.Warden, K.D.Thoben, et al:: An internet of things for transport logistics-an approach to connecting the information and material flows in autonomous cooperating logistics processes(MITIP, 2010). 